\title{
Classic Biphasic Pulmonary Blastoma in an Adult Patient
}

\author{
Rajendra Kumar ${ }^{1}$, Vijay Raturi ${ }^{2}$, Manoj Jain ${ }^{3}$, Dewesh Kishan ${ }^{4}$, Madanlal Bhatt ${ }^{5}$
}

\begin{abstract}
Pulmonary blastoma (PB) is a disease of early childhood. We have reported here a case of classic biphasic pulmonary blastoma (CBPB) in a young adult patient. This is intended at highlighting the fact, that PB occurs in adult individuals, though not a very common occurrence. Thus, high index of suspicion with typically well-defined radiological images may help in clinching diagnosis, though histopathological examination with immunohistochemical evaluation may establish the diagnosis at an early stage, which may be crucial in successful management of such cases.
\end{abstract}

Keywords: Pulmonary blastoma, Computed tomography, Immunohistochemisty, Survival, Lung cancer

\section{Introduction}

Pulmonary blastoma (PB) is a malignant tumor of lung affecting children. $\mathrm{PB}$ is a rare entity having an incidence between $0.25-0.5 \%$ of all primary lung malignancies. PB occurs exclusively in children younger than 12 years. A 21year old healthy male, non-smoker was admitted in our hospital with complaint of chest pain, haemoptysis, dyspnea, generalized weakness and loss of weight. On Endobronchial biopsy of left lung mass and immunohistochemistry (IHC) patient was diagnosed with classic biphasic pulmonary blastoma. Patient is not having any significant history of medical illness in the past. Patient is not having family history of similar disease or any other malignancy in 1st and 2nddegree relatives.

\section{Future Scope}

- PB occurs in adult individuals as well, though not a very common occurrence.

- High index of suspicion with typically well-defined radiological image may help in clinching early diagnosis.

- Histopathological examination with immunohistochemical evaluation may establish the diagnosis at an early stage, which may be crucial in successful management of such cases.

I'm writing this case report, so that my case report can help and guide physicians in faster diagnosis and better management of the patients diagnosed with $\mathrm{PB}$ in future.

\section{Discussion}

Pulmonary blastoma (PB) incidence is $0.25-0.5 \%$ of all malignant lung neoplasm and is a rare primary lung malignancy [1]. Due to the histological similarity of the tumor tissue of PB with the fetal lung tissue, Barnard described pulmonary blastoma as -lungembryoma" in 1952,[1]. Till now More than hundred cases of pulmonary blastoma have been reported in literature,[2]. A 21year old healthy male, non-smoker was admitted in our hospital with complaint of chest pain, haemoptysis, dyspnea, generalized weakness and loss of weight. Patient is not having any significant history of medical illness in the past. At the time of presentation, X-ray of chest Posterior
Anterior view (PA view) was suggestive of well defined round to oval homogeneous radio opacity noted in upper lobe of left lung (fig1). Computed tomography examination of thorax revealed a large well defined rounded in homogenously enhancing soft tissue space occupying lesion in left hilar and suprahilar region with extensions and mass effect (fig 2, 3, 4, 5). Laboratory investigations showed a leucocyte count of $6800 / \mathrm{mm} 3$, haemoglobin level of $10.1 \mathrm{~g} / \mathrm{dL}$, platelet count of 1.5 lac cells/mm3, serum AFP - 2.79ng/ml, serum LDH 547.3IU/L, serum beta- HCG $-<1.0 \mathrm{IU} / \mathrm{ml}$. Biopsy from left lung mass was taken from endobronchial route, which was histopathologicallysuggestive of PB (fig 6,7,8). Immunohistochemistry revealed cytokeratin and vimentin positivity (fig 9, 10, 11). Workup for metastasis of the disease was negative. Organ function evaluation like kidney function test (KFT), liver function test (LFT), electrocardiogram (ECG) and echocardiography (2DECHO) was done and was found to be within normal limits.

PB is further classified into three different subtypes based on the tissue component:

A. Monophasic pulmonary blastoma having only epithelial malignant tissue component and it's also known as well differentiated fetal adenocarcinoma (WDFA)

B. Classic biphasic pulmonary blastoma (CBPB) having both the mesenchymal and epithelial malignant tissue component.

C. Pleuropulmonaryblastoma (PPB) having only mesenchymal malignant tissue component.

CBPB is most common out of the above mentioned three subtypes,[2]. PPB is consider as an entirely different entity, and is referred to as the pulmonary blastoma of childhood,[3], whereas WDFA and CBPB commonly occurs in an adult individuals,[4]. Since 1999, the World health organization (WHO) classification has separated CBPB from the other two types of PB (WDFA and PPB) where it's now consider as a variant of sarcomatoid carcinomas,[5]. PB is commonly seen in the fourth decade of life, and it has a strong female preponderance,[6]. Our patient, a 21 years old healthy, young male, non smoker 


\section{International Journal of Science and Research (IJSR) \\ ISSN (Online): 2319-7064 \\ Index Copernicus Value (2013): 6.14 | Impact Factor (2014): 5.611}

presented with symptoms of chest pain, haemoptysis, dyspnea, and weight loss.

CBPB can occur at any age from birth to seventy years, and out of which $80 \%$ of CBPB occurs in an adult individual,[7]. Unlike WDFA and PPB, CBPB is common among the smokers, and it has slight male preponderance,[7]. Symptoms in CBPB are more like an URTI (Upper respiratory tract infection), which require investigations if the symptoms persist, or if it reoccurs. In CBPB, chest X-ray (posterior anterior view) and computed tomography (CT) of thorax is usually helpful and it typically shows a well-defined peripheral or midlung mass,[7]. Immunohistochemistry (IHC) plays a very important role in clinching the diagnosis of CBPB. An endobronchial biopsy from left lung mass was taken in our patient which was histopathologicallysuggestive of CBPB (fig 6,7,8). IHC revealed vimetin and cytokeratin positivity (fig 9,10,11). Workup for metastasis of the disease was negative. Organ function evaluation like kidney function test (KFT), liver function test (LFT), electrocardiogram (ECG) and echocardiography (2DECHO) was done and was found to be within normal limits.

The mainstay of treatment in PB is surgical excision of the tumor with negative margin, though the recurrence rate is quite high even after the resection of the tumor. Adjuvant treatment like post operative chemotherapy and radiotherapy can be quite useful to prolong the patient's survival, but the efficacy of this adjuvant treatment (post operative chemotherapy and radiotherapy) is not yet established and the literature is quite inadequate,[4]. Various chemotherapeutic agents in different combination regimes have been tried as neo-adjuvant and adjuvant treatment,[6, 7].

Due to extensive and inoperable disease, our patient received neo-adjuvant chemotherapy consisting of Ifosphamide and Adriamycin (IE) combination to downstage the tumor. A total of 4 cycles of this combination IE chemotherapy was given to the patient. Toxicities of the above mentioned IE regimen consisted of Grade 2/3 leukopenia during chemotherapy, which were managed successfully. No other hematological, cardiac or urinary toxicity was noted in this patient. Following which, patient was considered for surgery and he underwent left upper lobectomy. Subsequently Patient was given two more cycles of adjuvant chemotherapy comprising of same combination. The factors which point towards the unfavorable prognosis of the patient with $\mathrm{PB}$ are the size of tumor more than $5 \mathrm{~cm}$, recurrence of the tumor, biphasic type on IHC, metastatic disease at the time of presentation, and the frequent lymph node involvement,[1, 2]. In our patient, on the basis of the presence of two important prognostic factors the biphasic pattern of the disease on IHC and the large size of the tumor on X-ray chest (PA view) and computed tomography of thorax, patient was planned for adjuvant chemotherapy following resection of the tumor. Although the long term survival of the patient with $\mathrm{PB}$ have been reported in several individual case reports, but the overall prognosis of the patient with $\mathrm{PB}$ remain to be poor,[8]. In one survey of 83 patients with $\mathrm{PB}$, approximately mean survival of 33 months was reported in those patients where negative margin was achieved during surgical excision of the tumor, [8].

\section{References}

[1] C. S. Cutler, R. P. Michel, M. Yassa, and A. Langleben, - Plmonary blastoma: case report of a patient with a 7 year remission and review of chemotherapy experience in the world literature," Cancer, vol. 82, no. 3, pp. 462-467, 1998.

[2] M. N. Koss, L. Hochholzer, and T. O'Leary, -Plmonary blastomas," Cancer, vol. 67, no. 9, pp. 2368-2381, 1991.

[3] Manivel JC, Priest JR, Watterson J, et al. Pleuropulmonaryblastoma. The so-called pulmonary blastoma of childhood. Cancer. 1988; 62: 1516-26, http://dx.doi.org/10.1002/10970142(19881015)62:8<1516::AIDCNCR2820620812>3.0.CO;2-3

[4] Van Loo S, Boeykens E, Stappaerts I, et al. Classic biphasic pulmonary blastoma: a case report and review of the literature. Lung Cancer. 2011; 73: 127-32. PMid: 21513998. http://dx.doi.org/10.1016/j.lungcan.2011.03.018

[5] Travis WD, Brambilla E, Muller-Hermelink HK, et al. World Health Organization Classification of Tumours. Pathology and Genetics of Tumours of the Lung, Pleura, Thymus and Heart. Lyon: IARC Press; 2004.

[6] J. Robert, J. C. Pache, Y. Seium, M. De Perrot, and A. Spiliopoulos, - Plmonary blastoma: report of five cases and identification of clinical features suggestive of the disease," European Journal of Cardio-thoracic Surgery, vol. 22, no. 5, pp. 708-711, 2002.

[7] R. I. Walker, K. Suvarna, and S. Matthews, -Plmonary blastoma: presentation of two atypical cases and review of the literature," British Journal of Radiology, vol. 78, no. 929, pp. 437-440, 2005.

[8] R. K. P. Adluri, S. R. Boddu, A. Martin-Ucar, J. P. Duffy, F. D. Beggs, and W. E. Morgan, -Plmonary blastoma-a rare tumor with variable presentation," European Journal of Cardio-thoracic Surgery, vol. 29, no. 2, pp. 236-239, 2006.

\section{Figure Captions}

Figure 1: Well defined round to oval homogeneous radioopacity is noted in upper lobe of left lung

Figure 2 Fig 3 Fig 4 Fig 5 : Well defined round to oval heterogenous soft tissue attenuating mass is noted in apicoposterior segment of left upper lobe with extension into apical segment of left lower lobe

Figure 6: Tumor bits lined by cubo-columnar epithelial cells with focal Pseudostratification

Figure 7: The mesenchymal cells show round to spindle shaped hyperchromatic nuclei, inconspicuous nucleoli and indistinct cytoplasm.

Figure 8: Interspersed areas of necrosis. 


\section{International Journal of Science and Research (IJSR) \\ ISSN (Online): 2319-7064 \\ Index Copernicus Value (2013): 6.14 | Impact Factor (2014): 5.611}

Figure 9: The lining epithelial cells are positive for cytokeratin while the Mesenchymalcells are negative (X200 cytokeratin).

Figure 10: The lining epithelial cells are positive for cytokeratin while the Mesenchymal cells are negative(X400 cytokeratin).

Figure 11: The mesenchymal cells show immunopositivity for vimentin

\section{Author Profile}

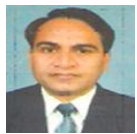

Dr. Madan lal Bhatt is Head of Department, Department of Radiotherapy, King George Medical University, Lucknow, India

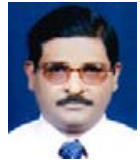

Dr. Rajendra Kumar is Associate Professor, Department of Radiotherapy, King George Medical University, Lucknow, India

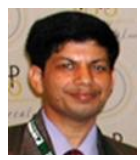

Dr. Manoj Jain is Professor, Department of Pathology, Sanjay Gandhi Post Graduate Institute, Lucknow, India

Dr. Vijay Raturi is Junior resident, Department of Radiotherapy, King George Medical University, Lucknow, India

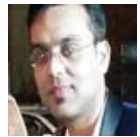

Dr Dewesh Kishan is Junior Resident, Department of Radiotherapy, King George Medical University, Lucknow, India

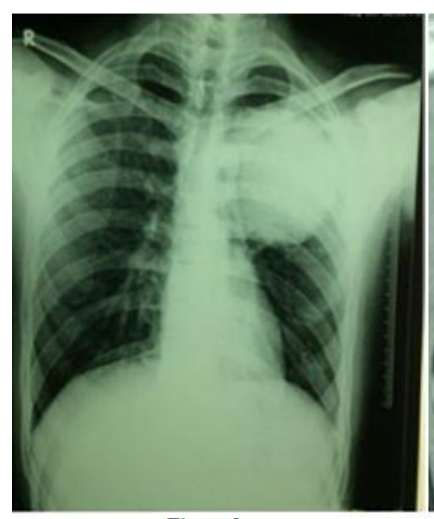

Figure 1

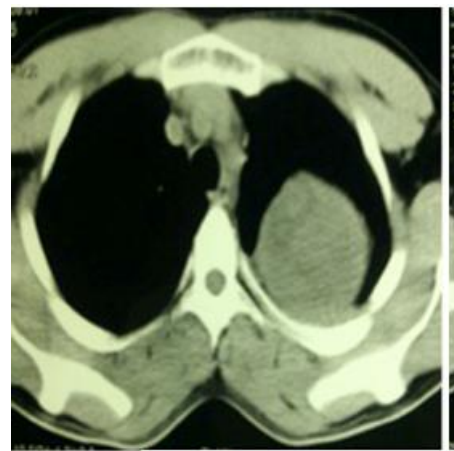

Figure 3
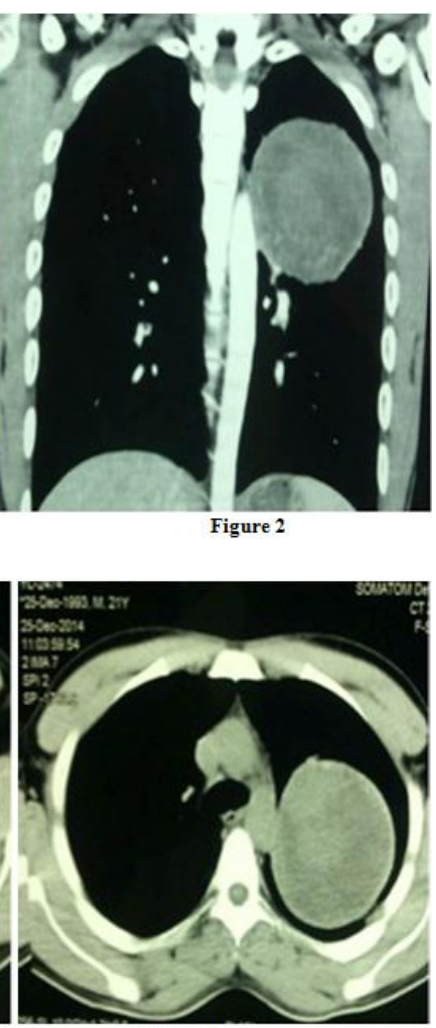
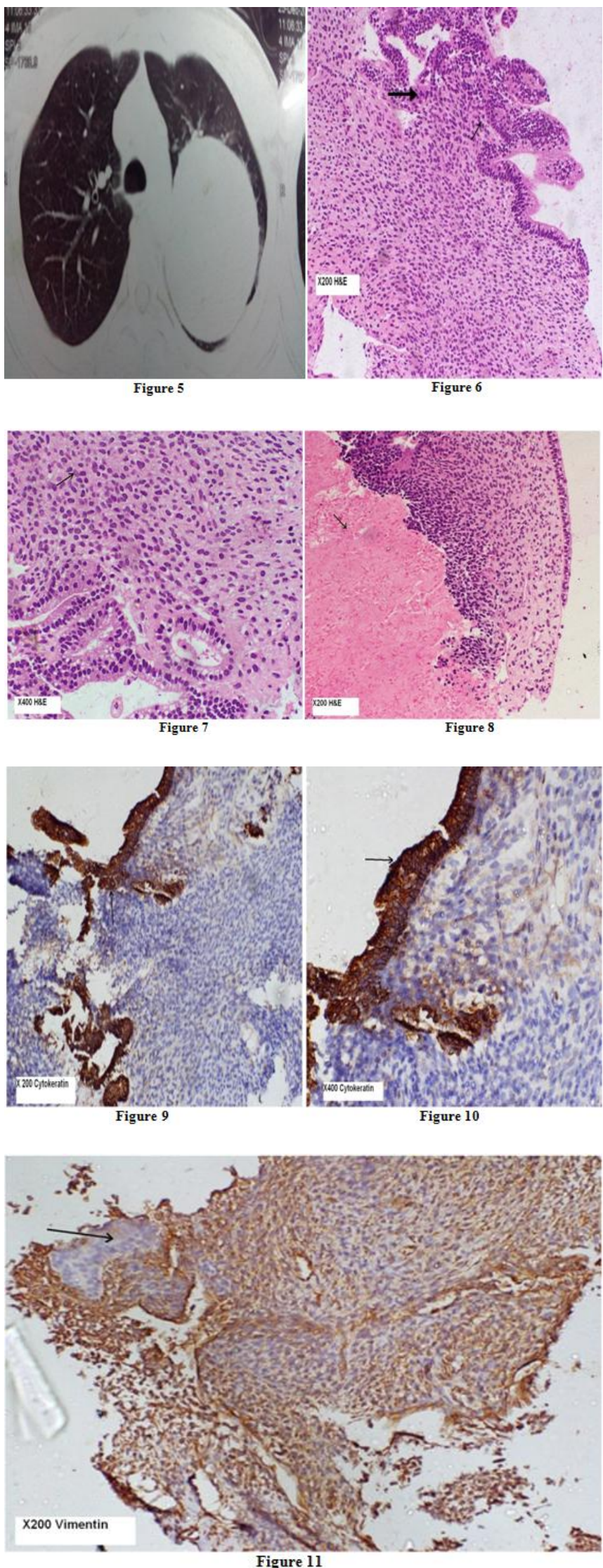